\title{
The Distribution of Small Gaps Between Successive Primes
}

\author{
By Richard P. Brent
}

\begin{abstract}
For $r \geqq 1$ and large $N$, a well-known conjecture of Hardy and Littlewood implies that the number of primes $p \leqq N$ such that $p+2 r$ is the least prime greater than $p$ is asymptotic to

$$
\int_{2}^{N}\left(\sum_{k=1}^{r} \frac{A_{r, k}}{(\log x)^{k+1}}\right) d x
$$

where the $A_{r, k}$ are certain constants. We describe a method for computing these constants. Related constants are given to 10D for $r=1(1) 40$, and some empirical evidence supporting the conjecture is mentioned.
\end{abstract}

1. Introduction. Let $r$ be a fixed positive integer and $N$ a large integer. Hardy and Littlewood [4] conjectured that the number of primes $p \leqq N$ such that $p+2 r$ is also prime is

$$
P_{N}(r) \sim A_{r, 1} \int_{2}^{N} \frac{d x}{(\log x)^{2}}
$$

where

$$
\begin{aligned}
A_{r, 1} & =2 c_{2} \prod_{\left.q\right|_{r}} \frac{q-1}{q-2}, \\
c_{2} & =\prod_{q} \frac{1-2 / q}{(1-1 / q)^{2}}=0.66016 \cdots
\end{aligned}
$$

is the "twin-prime" constant, and $q$ runs over the odd primes (this convention is adopted throughout). Conjecture (1) has been substantiated empirically and extended by several authors, e.g. [1], [3], [5] and [6].

In this paper, we study the number $Q_{N}(r)$ of primes $p \leqq N$ such that $p+2 r$ is the first prime after $p$ (so $p+1, p+2, \cdots, p+2 r-1$ are composite). In Section 2, we use the principle of inclusion and exclusion to deduce from a conjecture of Hardy and Littlewood that

$$
Q_{N}(r) \sim \int_{2}^{N}\left(\sum_{k=1}^{r} \frac{A_{r, k}}{(\log x)^{k+1}}\right) d x,
$$

where the constants $A_{r, k}$ are defined by (8) below. Previously, $Q_{N}(r)$ seems to have been studied only for $r \leqq 4$, although the magnitude of the first prime $p$ followed by $2 r-1$ consecutive composite numbers has been investigated (see [2] and the references given there).

Received February 13, 1973.

AMS (MOS) subject classifications (1970). Primary 10-04, 10A20, 10A25, 10A40, 65A05.

Key words and phrases. Prime, distribution of primes, Hardy-Littlewood conjecture, prime gap, twin primes. 
In Section 3 we discuss the computation of the constants $A_{r, k}$, and related constants $B_{r, k}$ (see Eq. (12)) are given for $r \leqq 40$ in Table 1. Some empirical evidence for conjecture (4) is given in Section 4.

2. Derivation of the Conjecture. If $0<m_{1}<m_{2}<\cdots<m_{s}$, Hardy and Littlewood [4] conjecture that the number of primes $p \leqq N$ such that $p+2 m_{i}$ is prime for $i=1, \cdots, s$ is

$$
P_{N}\left(m_{1}, \cdots, m_{s}\right) \sim C\left(m_{1}, \cdots, m_{s}\right) \int_{2}^{N} \frac{d x}{(\log x)^{s+1}},
$$

where

$$
C\left(m_{1}, \cdots, m_{s}\right)=2^{s} \prod_{q}(1-1 / q)^{-(s+1)}\left(1-w_{\mathrm{m}}(q) / q\right)
$$

and $w_{\mathrm{m}}(q)$ is the number of distinct residues of $0, m_{1}, \cdots, m_{s}$ modulo the odd prime $q$. (Conjecture (1) is just the special case $s=1, m_{1}=r$ of (5).)

From the principle of inclusion and exclusion (see, e.g. [7]),

$$
Q_{N}(r)=\sum_{s=0}^{r-1}(-1)^{s} \sum_{0<m_{1}<\cdots<m_{s}<r} P_{N}\left(m_{1}, \cdots, m_{s}, r\right) .
$$

From (5), this gives the conjecture (4) if

$$
A_{r, k}=(-1)^{k+1} \sum_{0<m_{1}<\cdots<m_{k-1}<r} C\left(m_{1}, \cdots, m_{k-1}, r\right) .
$$

3. Computation of the Constants $A_{r, k}$. Since $w_{\mathrm{m}}(q)=s+1$ for all $q>m_{\mathrm{s}}$, $C\left(m_{1}, \cdots, m_{s}\right)$ may easily be found if the Hardy-Littlewood constants

$$
c_{k}=\prod_{a>k} \frac{1-k / q}{(1-1 / q)^{k}}
$$

are known. These may be evaluated by the method of Wrench [10] and Mayoh [8].

Thus, the computation of $A_{r, k}$ from (8) appears to be straightforward. However, the sum in (8) involves $\left(\begin{array}{c}r-1 \\ k-1\end{array}\right)$ terms, so the evaluation of $A_{r, 1}, \cdots, A_{r, r}$ in the obvious way requires the evaluation of $2^{r-1}$ terms $C\left(m_{1}, \cdots, m_{k-1}, r\right)$. We shall show how this may be reduced to the evaluation of $O\left(2^{2 r / 3}\right)$ terms.

It is easy to evaluate $A_{r, k}$ if the integer

$$
T_{r, k}=\sum_{0<m_{1}<} \prod_{i<m_{k-1}<r} \prod_{q \leqq r+1}\left(q-w_{\mathbf{m}, r}(q)\right)
$$

can be evaluated, where $w_{\mathrm{m}, r}(q)$ is the number of distinct residues of $0, m_{1}, \cdots, m_{k-1}$, $r$ modulo the odd prime $q$. By omitting all the terms with $w_{\mathrm{m}, r}(3)=3$, and using symmetry if $3 \mid r$, we find that

$$
T_{r, k}=K_{r} \sum_{0<m_{1}<\cdots<m_{k-1}<r: m_{i} \neq r^{\prime}(\bmod 3)} \prod_{j \leqq q \leqq r+1}\left(q-w_{\mathrm{m}, r}(q)\right),
$$

where 


$$
K_{r}=\left\{\begin{array}{ll}
1 & \text { if } r \not \equiv 0(\bmod 3) \\
2 & \text { if } r \equiv 0(\bmod 3)
\end{array}\right\},
$$

and

$$
r^{\prime}=\left\{\begin{array}{ll}
1 & \text { if } r \equiv 2(\bmod 3) \\
2 & \text { if } r \neq \equiv 2(\bmod 3)
\end{array}\right\} .
$$

Thus, we may compute $T_{r, k}$ by summing

$$
\left[\begin{array}{c}
\lfloor 2(r-1) / 3\rfloor \\
k-1
\end{array}\right] \text { instead of }\left[\begin{array}{l}
r-1 \\
k-1
\end{array}\right)
$$

terms. An additional factor of 2 can be saved by symmetry if $r$ is not divisible by 3 .

It is interesting to note that $T_{r, k}=0$ if $k$ is so large that a cluster of $k-1$ primes cannot lie between two large primes $p$ and $p+2 r$ (see [9]). Surprisingly, the least such $k$ is not a monotonic function of $r$, for $T_{10,6} \neq 0$ and $T_{11,6}=0$.

The constants $A_{r, k}$ for $r \leqq 40$ were found by computing the $T_{r, k}$ as suggested above. Since the $A_{r, k}$ vary greatly in size, it is more convenient to work with

$$
B_{r, k}=\left\{\begin{array}{ll}
A_{r, 1} & \text { if } k=1 \\
-A_{r, k} / A_{r, k-1} & \text { if } k>1 \text { and } A_{r, k-1} \neq 0 \\
0 & \text { if } k>1 \text { and } A_{r, k-1}=0
\end{array}\right\} .
$$

Thus, the integrand in (4) is $B_{r, 1} z^{2}\left(1-B_{r, 2} z\left(\cdots\left(1-B_{r, r} z\right) \cdots\right)\right)$, where $z=$ $1 /(\log x)$.

Table 1 gives the constants $B_{r, k}$, believed to be correctly rounded to $10 \mathrm{D}$, for $1 \leqq k \leqq r \leqq 40$. Values which are omitted are zero.

4. Empirical Evidence for the Conjecture. A "gap of length $2 r$ " is defined to be an interval $(p, p+2 r)$ where $p$ and $p+2 r$ are successive primes. The number of gaps of length $2,4, \cdots, 80$ in an interval $(M, N)$ was compared with the number predicted by conjecture (4) for various $M$ and $N$ in the range $\left(10^{6}, 10^{16}\right)$. (For example, $M=10^{6}, 10^{7}, \cdots, 10^{15}$ and $N=M+10^{6}$.) The actual and predicted gap distributions agreed closely in all the intervals considered. Detailed results have been deposited in the UMT file of this journal.

As a typical example, results for the interval $\left(10^{6}, 10^{9}\right)$ are given in Table 2 . For $r=1,2, \cdots, 40$, the table gives the actual number of gaps of length $2 r$, and the number predicted from

$$
\int_{10^{8}}^{10^{9}}\left(\sum_{k=1}^{r} \frac{A_{r, k}}{(\log x)^{k+1}}\right) d x .
$$

In $\left(10^{6}, 10^{9}\right)$, there are 50769035 gaps, and

$$
\int_{11^{8}}^{10^{9}} \frac{d x}{\log x} \bumpeq 50770607.4
$$


TABLE 1

The constants $B_{r, k}$ for $r=1(1) 40$ (values omitted are zero)

\begin{tabular}{|c|c|c|c|c|c|}
\hline$r$ & $\mathrm{k}$ & $\mathrm{B}_{\mathrm{r}, \mathrm{k}}$ & $r$ & $\mathrm{k}$ & $\mathrm{B}_{r, k}$ \\
\hline 1 & 1 & 1.3203236317 & \multirow[t]{3}{*}{11} & \multirow[t]{3}{*}{1} & \multirow{3}{*}{$\begin{array}{r}1.4670262574 \\
15.3430844044 \\
5.5503309283 \\
2.3653954168 \\
0.8429475787\end{array}$} \\
\hline 2 & 1 & 1.3203236317 & & & \\
\hline 3 & $\frac{1}{2}$ & $\begin{array}{l}2.6406472634 \\
2.1648090870\end{array}$ & & & \\
\hline 4 & $\begin{array}{l}1 \\
2 \\
3\end{array}$ & $\begin{array}{l}1.3203236317 \\
4.3296181741 \\
0.7261756149\end{array}$ & \multirow[t]{2}{*}{12} & $\begin{array}{l}1 \\
2 \\
3 \\
4 \\
5\end{array}$ & \multirow{2}{*}{$\begin{array}{r}2.6406472634 \\
18.4008772398 \\
7.0705502451 \\
3.3815938634 \\
1.6080940365 \\
0.6066697903\end{array}$} \\
\hline 5 & $\frac{1}{2}$ & $\begin{array}{l}1.7604315089 \\
4.8708204458\end{array}$ & & 6 & \\
\hline & 3 & 0.9682341532 & \multirow[t]{2}{*}{13} & \multirow{2}{*}{$\begin{array}{l}1 \\
2 \\
3 \\
4 \\
5 \\
6 \\
7\end{array}$} & \multirow{2}{*}{$\begin{array}{r}1.4403530528 \\
20.7370670462 \\
8.1550214623 \\
4.0633477298 \\
2.1093164691 \\
1.0254787648 \\
0.3885721249\end{array}$} \\
\hline 6 & $\begin{array}{l}1 \\
2 \\
3 \\
4\end{array}$ & $\begin{array}{l}2.6406472634 \\
7.5768318046 \\
2.0747874712 \\
0.4881403766\end{array}$ & & & \\
\hline 7 & $\begin{array}{l}1 \\
2 \\
3 \\
4\end{array}$ & $\begin{array}{l}1.5843883580 \\
9.0200378627 \\
2.7110556291 \\
0.7845113196\end{array}$ & \multirow[t]{2}{*}{14} & $\begin{array}{l}1 \\
2 \\
3\end{array}$ & \\
\hline 8 & $\begin{array}{l}1 \\
2 \\
3 \\
4\end{array}$ & $\begin{array}{r}1.3203236317 \\
10.8240454352 \\
3.4856429517 \\
1.2203509416\end{array}$ & & $\begin{array}{l}4 \\
5 \\
6 \\
7\end{array}$ & \\
\hline & 5 & 0.2845598501 & \multirow[t]{2}{*}{15} & \multirow{2}{*}{$\begin{array}{l}1 \\
2 \\
3 \\
4 \\
5 \\
6 \\
7 \\
8\end{array}$} & \multirow{2}{*}{$\begin{array}{r}3.5208630178 \\
24.4961678255 \\
9.9210862934 \\
5.1517209912 \\
2.8451655917 \\
1.5366370101 \\
0.7430478393 \\
0.2639864981\end{array}$} \\
\hline 9 & $\begin{array}{l}1 \\
2 \\
3 \\
4 \\
5\end{array}$ & $\begin{array}{r}2.6406472634 \\
12.4476522505 \\
4.2097137097 \\
1.6108632428 \\
0.4656433910\end{array}$ & & & \\
\hline 10 & $\begin{array}{l}1 \\
2 \\
3 \\
4 \\
5 \\
6\end{array}$ & $\begin{array}{r}1.7604315089 \\
15.8301664490 \\
5.7597518859 \\
2.5248640170 \\
1.0244154603 \\
0.2600013387\end{array}$ & 16 & $\begin{array}{l}1 \\
2 \\
3 \\
4 \\
5\end{array}$ & $\begin{array}{r}1.3203236317 \\
27.4930754054 \\
11.3952967179 \\
6.1214210653 \\
3.5632265426\end{array}$ \\
\hline
\end{tabular}


TABLE 1 (continued)

\begin{tabular}{|c|c|c|c|c|c|}
\hline$r$ & $\mathrm{k}$ & $\mathrm{B}_{\mathrm{r}, \mathrm{k}}$ & $r$ & $k$ & ${ }^{B} r, k$ \\
\hline \multirow[t]{2}{*}{16} & $\begin{array}{l}6 \\
7\end{array}$ & $\begin{array}{l}2.1006626920 \\
1.1950083565\end{array}$ & 20 & 10 & 0.1513663376 \\
\hline & $\begin{array}{l}8 \\
9\end{array}$ & $\begin{array}{l}0.6157145513 \\
0.2444874923\end{array}$ & \multirow[t]{2}{*}{21} & \multirow{2}{*}{$\begin{array}{r}1 \\
2 \\
3 \\
4 \\
5 \\
6 \\
7 \\
8 \\
9 \\
10 \\
11\end{array}$} & \multirow{2}{*}{$\begin{array}{r}3.1687767161 \\
36.3539740286 \\
15.6509822856 \\
8.8131644369 \\
5.4466534309 \\
3.4737488749 \\
2.2032572055 \\
1.3409356167 \\
0.7429532570 \\
0.3357930107 \\
0.0896365942\end{array}$} \\
\hline 17 & $\begin{array}{l}1 \\
2 \\
3 \\
4 \\
5 \\
6 \\
7 \\
8 \\
9\end{array}$ & $\begin{array}{r}1.4083452071 \\
27.7245360198 \\
11.4984742518 \\
6.1729997100 \\
3.5819776177 \\
2.0946244877 \\
1.1705496702 \\
0.5824231949 \\
0.2198886873\end{array}$ & & & \\
\hline 18 & $\begin{array}{r}1 \\
2 \\
3 \\
4 \\
5 \\
6 \\
7 \\
8 \\
9 \\
10\end{array}$ & $\begin{array}{r}2.6406472634 \\
30.0425246784 \\
12.6122048727 \\
6.8853036007 \\
4.0940729018 \\
2.4873794831 \\
1.4840852989 \\
0.8373344927 \\
0.4234899981 \\
0.1658772706\end{array}$ & 22 & $\begin{array}{r}1 \\
2 \\
3 \\
4 \\
5 \\
6 \\
7 \\
8 \\
9 \\
10 \\
11\end{array}$ & $\begin{array}{r}1.4670262574 \\
38.5221012010 \\
16.7436006742 \\
9.5502460845 \\
6.0071544989 \\
3.9277664665 \\
2.5837231927 \\
1.6635481779 \\
1.0125375871 \\
0.5464248161 \\
0.2174507624\end{array}$ \\
\hline 19 & $\begin{array}{l}1 \\
2 \\
3 \\
4 \\
5 \\
6 \\
7 \\
8 \\
9\end{array}$ & $\begin{array}{r}1.3979897277 \\
31.9331890432 \\
13.4561073779 \\
7.3677263519 \\
4.3825468884 \\
2.6448059150 \\
1.5377429643 \\
0.7999341181 \\
0.3061670870\end{array}$ & \multirow[t]{2}{*}{23} & $\begin{array}{r}1 \\
2 \\
3 \\
4 \\
5 \\
6 \\
7 \\
8 \\
9 \\
10\end{array}$ & $\begin{array}{r}1.3831961856 \\
39.9075175164 \\
17.3771078681 \\
9.9252611046 \\
6.2469956507 \\
4.0820403447 \\
2.6778338225 \\
1.7129792116 \\
1.0284516322 \\
0.5388263843\end{array}$ \\
\hline 20 & $\frac{1}{2}$ & $\begin{array}{r}1.7604315089 \\
33.0230029036\end{array}$ & & 11 & 0.199204519 \\
\hline & $\begin{array}{l}3 \\
4 \\
5 \\
6 \\
7 \\
8 \\
9\end{array}$ & $\begin{array}{r}14.0212494141 \\
7.7552428532 \\
4.6798483508 \\
2.8876803489 \\
1.7450477061 \\
0.9833612019 \\
0.4731308341\end{array}$ & 24 & $\begin{array}{l}1 \\
2 \\
3 \\
4 \\
5 \\
6 \\
7\end{array}$ & $\begin{array}{r}2.6406472634 \\
41.6087903720 \\
18.2211462744 \\
10.4857133121 \\
6.6675963762 \\
4.4204962521 \\
2.9630734223\end{array}$ \\
\hline
\end{tabular}


TABLE 1 (continued)

\begin{tabular}{|c|c|c|c|c|c|}
\hline$r$ & $k$ & ${ }^{B_{r, x}}$ & $r$ & $k$ & ${ }^{B} r, k$ \\
\hline \multirow[t]{2}{*}{24} & $\begin{array}{l}8 \\
9\end{array}$ & $\begin{array}{l}1.9613859814 \\
1.2496708257\end{array}$ & 27 & 13 & 0.1729604958 \\
\hline & $\begin{array}{l}10 \\
11 \\
12\end{array}$ & $\begin{array}{l}0.7375923983 \\
0.3737970930 \\
0.1307666522\end{array}$ & \multirow[t]{2}{*}{28} & \multirow{2}{*}{$\begin{array}{l}1 \\
2 \\
3 \\
4 \\
5 \\
6 \\
7 \\
8 \\
9 \\
10 \\
11 \\
12 \\
13 \\
14\end{array}$} & \multirow{2}{*}{$\begin{array}{r}1.5343883580 \\
49.7793339545 \\
22.2516291007 \\
13.1317161092 \\
8.6175937443 \\
5.9494259004 \\
4.2078174539 \\
2.9992575087 \\
2.1274078880 \\
1.4833716834 \\
1.0014969256 \\
0.6388395411 \\
0.3646341816 \\
0.1558821625\end{array}$} \\
\hline 25 & $\begin{array}{r}1 \\
2 \\
3 \\
4 \\
5 \\
6 \\
7 \\
8 \\
9 \\
10 \\
11\end{array}$ & $\begin{array}{r}1.7504315089 \\
44.4259414830 \\
19.6478400115 \\
11.4435289614 \\
7.3859456123 \\
4.9901941363 \\
3.4285605747 \\
2.3470123849 \\
1.5694239568 \\
0.9991223480 \\
0.5797444199\end{array}$ & & & \\
\hline & $\begin{array}{l}12 \\
13\end{array}$ & $\begin{array}{l}0.2779751671 \\
0.0773583678\end{array}$ & \multirow[t]{2}{*}{29} & & \multirow{2}{*}{$\begin{array}{r}1.3692245069 \\
49.7351293969 \\
22.2010534736 \\
13.0749059856 \\
8.5538846663 \\
5.8779731882 \\
4.1277954713 \\
2.9100995690 \\
2.0292305179 \\
1.3777494229 \\
0.8927963590 \\
0.5361400447 \\
0.2829661211 \\
0.1112806842\end{array}$} \\
\hline 26 & $\begin{array}{r}1 \\
2 \\
3 \\
4 \\
5 \\
6 \\
7 \\
8 \\
9 \\
10 \\
11\end{array}$ & $\begin{array}{r}1.4403530528 \\
45.5513844929 \\
20.1021552452 \\
11.6727241289 \\
7.5016123702 \\
5.0370857637 \\
3.4292205066 \\
2.3145847402 \\
1.5125764268 \\
0.9245068977 \\
0.4938397071\end{array}$ & & & \\
\hline
\end{tabular}

$27 \quad 1 \quad 2.6406472634$ 47.3706617010 21.0944825152 12.3891416646 8.0793108307 5.5302801907 3.8641962264 2.7052770199 1.8661755593

$10 \quad 1.2435276562$

110.7763137851

120.4271256883

$\begin{array}{rr}1 & 3.5208630178 \\ 2 & 53.4568780418 \\ 3 & 24.0119086856 \\ 4 & 14.2465572306 \\ 5 & 9.4040165228 \\ 6 & 6.5333739503 \\ 7 & 4.6514933053 \\ 8 & 3.3376210785 \\ 9 & 2.3821089677 \\ 10 & 1.6694186696 \\ 11 & 1.1314474006 \\ 12 & 0.7264160913 \\ 13 & 0.4281428785\end{array}$


TABLE 1 (continued)

\begin{tabular}{|c|c|c|c|c|c|}
\hline$r$ & $\mathrm{k}$ & ${ }^{B} r, k$ & $r$ & $\mathrm{k}$ & ${ }^{B_{r, k}}$ \\
\hline 30 & $\begin{array}{l}14 \\
15\end{array}$ & $\begin{array}{l}0.2187972637 \\
0.0799007719\end{array}$ & \multirow[t]{2}{*}{33} & $\begin{array}{l}11 \\
12\end{array}$ & $\begin{array}{l}1.5642788142 \\
1.0853435243\end{array}$ \\
\hline \multirow[t]{2}{*}{31} & $\begin{array}{l}1 \\
2 \\
3 \\
4\end{array}$ & $\begin{array}{r}1.3658520328 \\
57.0564427867 \\
25.7883873035 \\
15.4134377102\end{array}$ & & $\begin{array}{l}13 \\
14 \\
15 \\
16\end{array}$ & $\begin{array}{l}0.1167404804 \\
0.4387634196 \\
0.2379412131 \\
0.1004264844\end{array}$ \\
\hline & $\begin{array}{r}5 \\
6 \\
7 \\
8 \\
9 \\
10 \\
11 \\
12 \\
13 \\
14 \\
15\end{array}$ & $\begin{array}{r}10.2643380469 \\
7.2079466964 \\
5.2002014685 \\
3.7939887877 \\
2.7661319314 \\
1.9930796451 \\
1.4012127006 \\
0.9442903513 \\
0.5922053004 \\
0.3249621929 \\
0.1294626296\end{array}$ & \multirow[t]{2}{*}{34} & $\begin{array}{r}1 \\
2 \\
3 \\
4 \\
5 \\
6 \\
7 \\
8 \\
9 \\
10 \\
11\end{array}$ & $\begin{array}{r}1.4083452071 \\
61.9919072207 \\
28.2142671194 \\
16.9990534791 \\
11.426543326 \\
8.1130495630 \\
5.9309308916 \\
4.3972048990 \\
3.2707071778 \\
2.4178440362 \\
1.7589282962\end{array}$ \\
\hline \multirow[t]{2}{*}{32} & $\begin{array}{l}1 \\
2 \\
3 \\
4 \\
5\end{array}$ & $\begin{array}{r}1.3203236317 \\
56.0765841791 \\
25.2955005318 \\
15.0822774906 \\
10.0134861920\end{array}$ & & $\begin{array}{l}12 \\
13 \\
14 \\
15 \\
16\end{array}$ & $\begin{array}{l}1.2438306194 \\
0.8398533041 \\
0.5252330428 \\
0.2854048569 \\
0.1108625403\end{array}$ \\
\hline & $\begin{array}{r}6 \\
7 \\
8 \\
9 \\
10 \\
11 \\
12 \\
13 \\
14 \\
15\end{array}$ & $\begin{array}{l}7.0047474948 \\
5.0282652653 \\
3.6439356759 \\
2.6321755336 \\
1.8716152565 \\
1.2903211212 \\
0.8438892387 \\
0.5049928034 \\
0.2585874366 \\
0.0987505835\end{array}$ & \multirow[t]{2}{*}{35} & $\begin{array}{r}1 \\
2 \\
3 \\
4 \\
5 \\
6 \\
7 \\
8 \\
9 \\
10\end{array}$ & $\begin{array}{r}2.1125178107 \\
62.7958185378 \\
28.6092743854 \\
17.2585563381 \\
11.6189657616 \\
8.2658057455 \\
6.0577632487 \\
4.5059818778 \\
3.3663563583 \\
2.5036711044\end{array}$ \\
\hline \multirow[t]{2}{*}{33} & $\begin{array}{l}1 \\
2 \\
3 \\
4 \\
5 \\
6 \\
7\end{array}$ & $\begin{array}{r}2.9340525149 \\
59.3116309805 \\
26.8938829613 \\
16.1336371765 \\
10.7902210471 \\
7.6158335959\end{array}$ & & $\begin{array}{l}11 \\
12 \\
13 \\
14 \\
15 \\
16 \\
17\end{array}$ & $\begin{array}{l}1.0313213153 \\
1.3167529951 \\
0.9093775214 \\
0.5943929626 \\
0.3596437584 \\
0.1989064249 \\
0.0993281933\end{array}$ \\
\hline & $\begin{array}{r}8 \\
9 \\
10\end{array}$ & $\begin{array}{l}2.0639911464 \\
2.9918723175 \\
2.1839746041\end{array}$ & 36 & $\begin{array}{l}1 \\
2 \\
3\end{array}$ & $\begin{array}{r}2.6406472634 \\
66.2111617092 \\
30.2766540599\end{array}$ \\
\hline
\end{tabular}


TABLE 1 (continued)

\begin{tabular}{|c|c|c|c|c|c|}
\hline$r$ & $\mathrm{k}$ & ${ }^{B_{r, k}}$ & $r$ & $k$ & ${ }^{B} r, k$ \\
\hline \multirow[t]{2}{*}{36} & $\begin{array}{r}4 \\
5 \\
6 \\
7 \\
8 \\
9 \\
10\end{array}$ & $\begin{array}{r}18.3409094425 \\
12.4068962334 \\
8.8754487506 \\
6.5470947651 \\
4.9080453851 \\
3.7017107962 \\
2.7858957162\end{array}$ & 38 & $\begin{array}{l}13 \\
14 \\
15 \\
16 \\
17 \\
18\end{array}$ & $\begin{array}{l}1.3131389130 \\
0.9365319820 \\
0.6404963934 \\
0.4138510165 \\
0.2463430835 \\
0.1194900846\end{array}$ \\
\hline & $\begin{array}{l}11 \\
12 \\
13 \\
14 \\
15 \\
16 \\
17\end{array}$ & $\begin{array}{l}2.0757081630 \\
1.5176476093 \\
1.0766924356 \\
0.7293724865 \\
0.4597244627 \\
0.2563308030 \\
0.1085109086\end{array}$ & \multirow[t]{2}{*}{39} & $\begin{array}{l}1 \\
2 \\
3 \\
4 \\
5 \\
6 \\
7\end{array}$ & $\begin{array}{r}2.8807061055 \\
70.6997065950 \\
32.5107597334 \\
19.8218855636 \\
13.509778123 \\
9.7499560283 \\
7.2677697292\end{array}$ \\
\hline \multirow[t]{2}{*}{37} & $\begin{array}{r}1 \\
2 \\
3 \\
4 \\
5 \\
6 \\
7 \\
8 \\
9 \\
10 \\
11\end{array}$ & $\begin{array}{r}1.3580471640 \\
66.9782027174 \\
30.6782419268 \\
18.6201628208 \\
12.6242292479 \\
9.0546344932 \\
6.6995839806 \\
5.0398868987 \\
3.8161157582 \\
2.8843429973 \\
2.1584604606\end{array}$ & & $\begin{array}{l}8 \\
9 \\
10 \\
11 \\
12 \\
13 \\
14 \\
15 \\
16 \\
17 \\
18\end{array}$ & $\begin{array}{l}5.5171282455 \\
4.2251859714 \\
3.2405820808 \\
2.4727472369 \\
1.8643285785 \\
1.3774162158 \\
0.9861397380 \\
0.6724449317 \\
0.4235592577 \\
0.2304613130 \\
0.0876237466\end{array}$ \\
\hline & $\begin{array}{l}11 \\
12 \\
13 \\
14 \\
15 \\
16 \\
17\end{array}$ & $\begin{array}{l}1.5839990281 \\
1.1250993393 \\
0.7576010703 \\
0.4653031637 \\
0.2382708022 \\
0.0741063531\end{array}$ & \multirow[t]{2}{*}{40} & \multirow{2}{*}{$\begin{array}{r}1 \\
2 \\
3 \\
4 \\
5 \\
6 \\
7 \\
8 \\
9 \\
10 \\
11 \\
12 \\
13 \\
14 \\
15 \\
16 \\
17 \\
18 \\
19\end{array}$} & \multirow{2}{*}{$\begin{array}{r}1.7604315089 \\
73.6107429917 \\
33.9328292881 \\
20.7460643325 \\
14.1837340795 \\
10.2726763283 \\
7.6886927466 \\
5.8644776442 \\
4.5166169192 \\
3.4879437671 \\
2.6845126281 \\
2.0469957441 \\
1.5364440188 \\
1.1267004833 \\
0.8001365673 \\
0.5450067101 \\
0.3529451420 \\
0.2139492394 \\
0.1062961616\end{array}$} \\
\hline 38 & $\begin{array}{r}1 \\
2 \\
3 \\
4 \\
5 \\
6 \\
7 \\
8 \\
9 \\
10 \\
11 \\
12\end{array}$ & $\begin{array}{r}1.3979897277 \\
69.4720528395 \\
31.9135466028 \\
19.4347144701 \\
13.2275487026 \\
9.5306429383 \\
7.0904008520 \\
5.3697954268 \\
4.1005791410 \\
3.1340488773 \\
2.3813915965 \\
1.7866244753\end{array}$ & & & \\
\hline
\end{tabular}


By summing (13) over $r=1, \cdots, 40$ and subtracting from (14), the predicted number of gaps of length greater than 80 is 473076.4 , and the actual number is 473186 .

Table 2 shows that (13) predicts quite well the number of gaps of various lengths in $\left(10^{6}, 10^{9}\right)$. Although the right sides of (1) and (4) are asymptotically equal, the higher terms in (4) are important for approximating $Q_{N}(r)$. It is interesting to note that in $\left(10^{6}, 10^{9}\right)$ there are less gaps (observed and predicted) for $r=31$ than for $r=32$, although $A_{31,1}=1.36 \cdots>A_{32,1}=1.32 \cdots$. Thus, the higher terms in (13) are significant.

TABLE 2

Actual and predicted gap distribution in $\left(10^{6}, 10^{9}\right)$

\begin{tabular}{rrrrrr}
$r$ & Actual & Predicted & $r$ & Actual & Predicted \\
\hline 1 & 3416337 & 3417060.1 & 21 & 953980 & 954689.0 \\
2 & 3416536 & 3417060.1 & 22 & 389432 & 389057.1 \\
3 & 6076242 & 6077407.1 & 23 & 334565 & 335337.0 \\
4 & 2689540 & 2688560.2 & 24 & 577051 & 577898.6 \\
5 & 3477688 & 3477436.8 & 25 & 327960 & 327323.5 \\
6 & 4460952 & 4460654.7 & 26 & 245727 & 245799.1 \\
7 & 2460332 & 2461360.3 & 27 & 410614 & 410578.1 \\
8 & 1843216 & 1842845.7 & 28 & 211409 & 211469.0 \\
9 & 3346123 & 3347229.6 & 29 & 181894 & 182398.0 \\
10 & 1821641 & 1823424.2 & 30 & 371743 & 372007.3 \\
11 & 1567507 & 1567220.8 & 31 & 115542 & 115837.8 \\
12 & 2364792 & 2362746.8 & 32 & 118927 & 118681.6 \\
13 & 1118410 & 1118419.0 & 33 & 216739 & 216467.5 \\
14 & 1218009 & 1218441.9 & 34 & 88383 & 88116.0 \\
15 & 2176077 & 2176130.5 & 35 & 125542 & 125688.7 \\
16 & 683346 & 682871.2 & 36 & 126650 & 126786.7 \\
17 & 718974 & 718118.6 & 37 & 62514 & 62578.8 \\
18 & 1170757 & 1169307.2 & 38 & 55107 & 55325.4 \\
19 & 548416 & 547688.6 & 39 & 105300 & 105390.3 \\
\hline 1048356 & 648539.8 & 40 & 53519 & 53578.4
\end{tabular}


Computer Centre

Australian National University

Canberra, Australia

1. P. T. Bateman \& R. A. HoRn, "A heuristic asymptotic formula concerning the distribution of prime numbers," Math Comp., v. 16, 1962, pp. 363-367. MR 26 \#6139.

2. R. P. BRENT, "The first occurrence of large gaps between successive primes," Math. Comp., v. 27, 1973, pp. 959-963.

3. F. Gruenberger \& G. Armerding, Statistics on the First Six Million Prime Numbers, Paper P-2460, The RAND Corporation, Santa Monica, Calif., 1961, 145 pp. (Copy deposited in the UMT File and reviewed in Math. Comp., v. 19, 1965, pp. 503-505.)

4. G. H. HARdY \& J. E. LitTlewood, "Some problems of 'partitio numerorum'; III: On the expression of a number as a sum of primes," Acta Math., v. 44, 1923, pp. 1-70.

5. M. F. Jones, M. Lal \& W. J. Blundon, "Statistics on certain large primes," Math. Comp., v. 21, 1967, pp. 103-107. (Corrigenda, v. 22, 1968, pp. 474 \& 911.) MR 36 \#3707.

6. D. H. LEHMER, "Tables concerning the distribution of primes up to 37 millions", 1957. Copy deposited in the UMT File and reviewed in $M T A C$, v. 13, 1959, pp. 56-57.

7. C. L. LiU, Introduction to Combinatorial Mathematics, McGraw-Hill, New York, 1968, Chap. 4. MR 38 \#3154.

8. В. Н. МАуОН, "The second Goldbach conjecture revisited," Nordisk Tidskr. Informationsbehandling $(B I T)$, v. 8, 1968, pp. 128-133. MR $39 \# 125$.

9. H. RIESEL, "Primes forming arithmetic series and clusters of large primes," Nordisk Tidskr. Informationsbehandling (BIT), v. 10, 1970, pp. 333-342. MR 44 \#65.

10. J. W. WRENCH, "Evaluation of Artin's constant and the twin-prime constant," Math. Comp., v. 15, 1961, pp. 396-398. MR 23 \# A1619. 